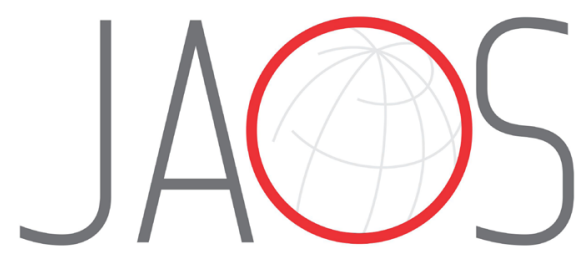
JOURNAL OF APPLIED ORAL SCIENCE

\title{
Prevalence of symptoms of
} temporomandibular disorders, oral behaviors, anxiety, and depression in Dentistry students during the period of social isolation due to COVID-19

\section{Abstract}

Rodrigo Antonio de MEDEIROS

Danielle Leal VIEIRA ${ }^{1}$

Emily Vivianne Freitas da SILVA ${ }^{2}$

Liliana Vicente Melo de Lucas

REZENDE ${ }^{1}$

Rodrigo Wendel dos SANTOS ${ }^{3}$ Lucas Fernando TABATA ${ }^{1}$
Submitted: June 5, 2020 Modification: August 17, 2020 Accepted: September 5, 2020

Corresponding address: Rodrigo Antonio de Medeiros Universidade de Brasília - Faculdade de Ciências da Saúde - Departamento de Odontologia - UnB Campus Darcy Ribeiro - 70910-900 Brasília - Distrito Federal - Brasil. Phone: 55-61-31071802 e-mail: rodrigomedeiros88@hotmail.com
Temporomandibular dysfunction (TMD), anxiety, and depression are disorders that, due to the current lifestyle, are affecting an increasing portion of the population. Investigating the prevalence of the symptoms of these disorders during the quarantine due to the coronavirus 2019 pandemic (COVID-19) is important to outline clinical strategies for patient care. Objective: This study assessed the prevalence of TMD symptoms, anxiety, depression, and oral behaviors and their associations during the social isolation due to COVID-19. Methodology: Questionnaires were used to assess TMD symptoms in accordance with the Diagnostic Criteria for Temporomandibular Disorders: clinical protocol and assessment instruments, a questionnaire to verify oral behaviors and Hospital Anxiety and Depression Scale to assess symptoms of anxiety and depression in students of dentistry at the Faculty of Health Sciences of the University of Brasília in May 2020. Qualitative data were subjected to descriptive statistics and chi-squared analysis $(p<0.05)$. The relationship between quantitative and qualitative data was evaluated using Spearman's rho correlation $(p<0.05)$. Results: There was a high prevalence of TMD symptoms, anxiety, and depression in the participants, resulting in association between gender and anxiety symptoms $(p=0.029)$. There was a positive correlation between oral behaviors and TMD symptoms $(r=0.364 ; p<0.001)$, between oral behaviors and anxiety symptoms $(r=0.312 ; p=0.001)$, and between oral behaviors and symptoms of depression $(r=0.216 ; p=0.021)$. Conclusion: Social isolation due to the COVID-19 pandemic has an impact on the prevalence of TMD symptoms, anxiety, and depression.

Keywords: Temporomandibular Joint Dysfunction Syndrome. Anxiety. Anxiety disorders. Myalgia. COVID-19.

${ }^{1}$ Universidade de Brasília, Faculdade de Ciências da Saúde, Departamento de Odontologia, Brasília, Distrito Federal, Brasil.

${ }^{2}$ Universidade Estadual Paulista, Faculdade de Odontologia de Araçatuba, Departamento de Materiais Odontológicos e Prótese, Araçatuba, São Paulo, Brasil.

${ }^{3}$ Dentista em clínica privada, Brasília, Distrito Federal, Brasil. 


\section{Introduction}

COVID-19 (coronavirus disease 2019) quickly spread in late January 2020 and attracted enormous attention worldwide. ${ }^{1}$ The new disease caused by the novel coronavirus (SARS-CoV-2) originated from a market in Wuhan, China, in early December 2019.2 On January 7, 2020, the Chinese Center for Disease Control and Prevention identified and isolated this new coronavirus, calling it severe acute respiratory syndrome coronavirus 2 (SARS-CoV-2). SARS-CoV-2 can be transmitted from one person to another through close contact, leading to COVID-19. ${ }^{3}$ Infected patients can develop severe respiratory distress, requiring intensive care, and can be fatal. ${ }^{1,3}$ Owing to this scenario, several countries in the world are adopting restrictive quarantine measures to control the spread of the virus and the collapse of health systems.

It is not uncommon for people with a confirmed diagnosis or with suspected COVID-19 to experience great psychological pressure. Health professionals are also susceptible to these problems, as they must care for infected patients, to decrease or, in some cases, to restrict living with their family, among other factors. ${ }^{4}$ In addition, people who are quarantined, fulfilling social isolation, restricted to leave, concerned about infection, afraid of death, lack information, and who have lost daily social relationships, can further experience high levels of anxiety and depression. ${ }^{5}$

Psychological factors are associated with the development of some diseases and disorders, including temporomandibular disorders (TMD), which is a collective term used to describe disorders related to temporomandibular joints (TMJs) and masticatory muscles, which are primarily responsible for the movement of TMJs and related structures. ${ }^{6}$ The etiology of TMD is multifactorial, including the combined action of environmental, biological, psychological, biomechanical, and neuromuscular factors. ${ }^{6,7}$ Symptoms are generally jaw pain, ear pain, toothache (of non-dental origin), joint pain, headache, and mandibular functional limitation. ${ }^{8,9}$ More than $50 \%$ of the population present some level of TMD, but only $3.6 \%$ to $7 \%$ require treatment. ${ }^{10}$ The highest prevalence is in women aged 20 to 40 years. ${ }^{10}$

Currently, the bio-psychosocial model studies the etiology of TMD due to biological factors, such as genetic or biochemical factors, psychological factors, such as anxiety, stress, and depression, and social factors, such as culture, family behavior, and socioeconomic status. However, the mechanisms by which psychological factors influence the development of TMD remain unknown. ${ }^{7}$

Therefore, studies that assess the influence of the COVID-19 pandemic, and its consequences on TMDs are important. Thus, the objective of this study was to assess the prevalence of TMD symptoms, anxiety, depression, and oral behaviors and their associations during the social isolation due to COVID-19. The null hypothesis is that there will be no association between TMD symptoms, anxiety, depression, and oral behaviors.

\section{Methodology}

This was a cross-sectional study performed on dental medicine students from the School of Health Sciences of the University of Brasília (FS/UnB). The study was approved by the National Council of Ethics in Research (CAAE: 30637620.2.0000.0008).

A minimum sample size of 104 participants was determined based on the size of the student population of this course of 220 students, an accuracy of $5 \%$, an estimated prevalence of $15 \%$, and a $95 \%$ confidence interval, using an online sample size calculator. (http:// sampsize.sourceforge.net/iface). All students of both genders were invited to participate in the research. All subjects signed an informed consent form before participation. Students on medication for anxiety and/ or depression were excluded.

Due to the impossibility of clinical evaluation because of the social isolation due to COVID-19, the evaluation of TMD symptoms, oral behaviors, anxiety, and depression was carried out through questionnaires, applied between May 12 and 19, 2020, and made available through a QR code and link, which was sent to class e-mails and disseminated on social networks.

General data such as name, age, gender, and medication use were collected. In addition, the following was asked:

How is your isolation going? Total social isolation - without ever leaving home; Partial social isolation - at home, but going to the market, pharmacy, and other essential services, or; No isolation - I am not performing social isolation.

Are you carrying out any physical activity at home? 
Yes or no.

\section{Assessment of the presence of TMD symptoms}

The Diagnostic Criteria for Temporomandibular Disorders: clinical protocol and assessment instruments (DC/TMD) questionnaire ${ }^{11}$ was translated and validated into Brazilian Portuguese and self-administered with objective questions about TMD symptoms. The answered questionnaires were analyzed by a specialist in the field and the participants were categorized into three groups according to their symptoms in the last 30 days: without TMD, with non-painful TMD, or painful TMD. For this, they were classified as either having painful TMD, based on the first two questions of the questionnaire related to the presence of pain and headache, or having non-painful TMD, based on questions related to the presence of joint sounds, closed locking, and/or open locking.

\section{Assessment of the presence of parafunctional habits/oral behaviors}

The DC/TMD oral behavior checklist (OBC) questionnaire, ${ }^{11}$ translated into and validated for Brazilian Portuguese, was used for self-completion, containing objective questions about the frequency of oral behaviors during sleep and during waking. For each $\mathrm{OBC}$ item, the participant was asked to report the frequency of occurrence in the last 30 days, using the options "none of the times" (0), "a small part of the time" (1), "some part of the time" (2), "most of the time" (3), "all the time" (4) for activities during awake hours, and "none of the time" $(0)$, " $<1$ night/month" (1), "1-3 nights/month" (2), "1-3 nights/week" (3), "4-7" nights/week" (4) for activities during sleeping hours. The values were added and assigned to each participant, where the higher the value, the more oral behaviors the individual would present.

\section{Assessment of anxiety and depression}

The Hospital Anxiety and Depression Scale (HADS) was used to assess symptoms of anxiety and depression. It is made of 14 items, 7 of which assess anxiety (HADS-A) and 7 depression (HADS-D). ${ }^{12}$ Each of its items can be scored from 0 to 3 , making up a maximum score of 21 points for each scale. ${ }^{13}$

Responses to the HADS items were obtained to assess frequency of anxiety and depression. The recommended cut-off points were adopted for both subscales: ${ }^{14}$

HAD-anxiety: no anxiety: 0 to 7; with anxiety: $\geq 8$.
HAD-depression: no depression: 0 to 7; with depression: $\geq 8$.

\section{Data analysis}

The results were tabulated and subjected to statistical analysis. Statistical analysis was performed using SPSS software (version 24.0, SPSS Inc., Chicago, USA). Descriptive statistics were used to analyze the demographic data of the participants included in the study. The normality analysis was performed using the Shapiro-Wilk test, with no normality for OBC values. Spearman's rho correlation was performed to verify the relationship between OBC values and TMD symptoms, anxiety symptoms, and depression symptoms. The Chi-Squared analysis was performed to verify the association between sex and TMD symptoms, gender and anxiety symptoms, gender and depression symptoms, isolation and TMD symptoms, isolation and anxiety symptoms, isolation and depression symptoms, physical activity and TMD symptoms, physical activity and anxiety symptoms, physical activity and depression symptoms, TMD symptoms and anxiety symptoms, and TMD symptoms and depression symptoms. All analyses were performed with a $5 \%$ level of significance.

\section{Results}

A total of 147 students started the process of filling the questionnaires, 127 of whom completed the questionnaire. Fourteen students were excluded, leaving 113 valid questionnaires. Of the total participants, 87 were female (77\%) and 26 were male $(23 \%)$, with a mean age of $21.46 \pm 2.37$ years.

Of the 113 participants, 87 practiced partial social isolation $(77 \%), 24$ practiced total social isolation $(21.2 \%)$, and 2 did not practice social isolation (1.8\%). Regarding physical activity, 54 participants did not perform activities (47.8\%) and 59 participants exercised at home (52.2\%).

A total of 51 participants had no symptoms of TMD in the last 30 days (45.2\%), 31 had symptoms of non-painful TMD (27.4\%), and 31 had symptoms of painful TMD (27.4\%). Fifty-seven participants had no anxiety symptoms, (50.4\%), of which 56 had anxiety symptoms (49.6\%) in the last 30 days. Four participants received TMD treatment before the COVID-19 pandemic, of which 3 had symptoms of 
Table 1- Frequency of study participants regarding the association between TMD symptoms and different analysis variables (Chi-squared, $p<0.05$ )

\begin{tabular}{|c|c|c|c|c|c|}
\hline Variables & & No TMD & Non-painful TMD & Painful TMD & P-value \\
\hline \multirow{2}{*}{ Gender } & Male & 10 & 11 & 5 & 0.143 \\
\hline & Female & 41 & 20 & 26 & \\
\hline \multirow{2}{*}{ Anxiety } & With symptoms & 22 & 15 & 19 & 0.277 \\
\hline & Without symptoms & 29 & 16 & 12 & \\
\hline \multirow{2}{*}{ Depression } & With symptoms & 18 & 13 & 13 & 0.771 \\
\hline & Without symptoms & 33 & 18 & 18 & \\
\hline \multirow{3}{*}{ Isolation } & Parcial isolation & 39 & 24 & 24 & 0.758 \\
\hline & Full isolation & 12 & 6 & 6 & \\
\hline & Nol isolation & 0 & 1 & 1 & \\
\hline \multirow[t]{2}{*}{ Physical activity } & Yes & 31 & 13 & 15 & 0.224 \\
\hline & No & 20 & 18 & 16 & \\
\hline
\end{tabular}

Table 2- Frequency of study participants regarding the association between anxiety symptoms and different analysis variables (Chisquared, $p<0.05)$

\begin{tabular}{ccccc}
\hline Variables & Wale & $\begin{array}{c}\text { With anxiety } \\
\text { symptoms }\end{array}$ & $\begin{array}{c}\text { Without anxiety } \\
\text { symptoms }\end{array}$ & P-value \\
\hline \multirow{2}{*}{ Gender } & Female & 8 & 18 & $0.029^{*}$ \\
\hline \multirow{2}{*}{ Isolation } & Parcial isolation & 48 & 39 & 0.166 \\
& Full isolation & 39 & 48 & 0.110 \\
\hline \multirow{2}{*}{ Physical activity } & Nol isolation & 16 & 1 & \\
\hline
\end{tabular}

Table 3- Frequency of study participants regarding the association between the symptoms of depression and different analysis variables (Chi-squared, $p<0.05$ )

\begin{tabular}{|c|c|c|c|c|}
\hline Variables & & $\begin{array}{c}\text { With depression } \\
\text { symptoms }\end{array}$ & $\begin{array}{c}\text { Without depression } \\
\text { symptoms }\end{array}$ & P-value \\
\hline \multirow{2}{*}{ Gender } & Male & 8 & 18 & 0.330 \\
\hline & Female & 36 & 51 & \\
\hline \multirow{3}{*}{ Isolation } & Parcial isolation & 32 & 55 & 0.686 \\
\hline & Full isolation & 11 & 13 & \\
\hline & Nol isolation & 1 & 1 & \\
\hline \multirow{2}{*}{ Physical activity } & Yes & 20 & 39 & 0.251 \\
\hline & No & 24 & 30 & \\
\hline
\end{tabular}

painful TMD in the last 30 days and 1 had no TMD symptom. Sixty-nine participants had no depression symptoms $(61.1 \%)$, while 44 had depression symptoms in the last 30 days (38.9\%).

No association was found between TMD symptomatology and gender, anxiety symptoms, depression symptoms, type of social isolation, and physical activity (Table 1 ). The association between anxiety symptoms and gender was verified $[X 2(2)=$ $4,769 ; p=0.029]$; however, there was no association between anxiety symptoms and type of social isolation and physical activity (Table 2 ). There was no association between depression symptoms and gender, type of social isolation, or physical activity (Table 3 ).

There was a positive correlation between oral behaviors (using the $O B C$ ) and TMD symptoms $(\rho=0.364$ and $p<0.001)$, between oral behaviors and anxiety symptoms $(\rho=0.312$ and $p=0.001)$, and between oral behaviors and symptoms of depression $(p=0.216$ and $p=0.021$ ) (Table 4). 
Table 4- Spearman's rho correlation between oral behavior value (OBC) and TMD symptoms, anxiety symptoms and depression symptoms $(p<0.05)$

\begin{tabular}{cc}
\hline $\begin{array}{c}\text { Spearman's rho } \\
\text { correlation }\end{array}$ & OBC \\
\hline TMD symptoms & Correlation coefficient $=0.364$ \\
& $p<0.001^{*}$ \\
\hline Anxiety symptoms & Correlation coefficient $=0.312$ \\
& $p=0.001^{*}$ \\
\hline Depression symptoms & Correlation coefficient $=0.216$ \\
& $\mathrm{p}=0.021^{*}$ \\
\hline
\end{tabular}

\section{Discussion}

The null hypothesis of the study was partially accepted since there was an association between gender and symptoms of anxiety and a correlation between oral behaviors and symptoms of TMD, anxiety, and depression. The first case of COVID-19 in Brazil was confirmed on February 26, in the city of São Paulo. With the emergence of new cases, Brasilia, located in the Federal District and capital of Brazil, had its first confirmed case on March 5, 2020, and there was a government decree demanding the interruption of classes and commercial activities from March 12, 2020, whereas on March 13 it was decided that classes should stop and academic calendar be suspended, starting on March 23. The dental medicine students of the University of Brasilia (UnB) started academic activities in 2020, and the academic calendar remains suspended until the present moment (May 30, 2020). This study was carried out 68 days after the decision to suspend academic activities, and all responses were influenced by the period of social isolation.

The results demonstrated a high rate of symptoms of anxiety and depression, at $49.6 \%$ and $38.9 \%$, respectively. A recent study carried out in China showed that $12.9 \%$ of the population affected by the quarantine showed symptoms of anxiety and $22.4 \%$ of depression. ${ }^{15} \mathrm{~A}$ recent review showed negative psychological effects, including post-traumatic stress, confusion, and anger, as a consequence of the longer quarantine duration, fear of infection, frustration, boredom, inadequate information, financial loss, restricted movement, and the loss of habitual routine and social contact have also been reported. ${ }^{5}$ In addition, regarding university students, there was a change in life and work planning due to the pandemic, with the possibility of graduation delay and uncertainties of the academic future. ${ }^{16} \mathrm{~A}$ recent study evaluated 44447 Chinese university students and found a prevalence of $7.7 \%$ of anxiety symptoms and $12.2 \%$ of depression symptoms. ${ }^{17}$ Another study performed on Spanish students showed rates of $34.2 \%$ for moderate to severe depression symptoms and $21.3 \%$ of anxiety symptoms. ${ }^{18}$ Due to this divergence of data, the influence of the pandemic on the psychological factors of university students is not yet known. Considering that the UnB adopted some measures of psychosocial help for students and civil servants, providing psychological assistance online and some extension projects of the School of Health Sciences were reformulated to help the academic and general population in the period of isolation. However, there is a need for wider dissemination of these services and the continuity of psychological assistance after the COVID-19 pandemic.

In the present study, there was a statistically significant association between gender and symptoms of anxiety, with $55.2 \%$ of women and $30.8 \%$ of men showing symptoms of anxiety. Anxiety symptoms are more common in women, especially among adolescents. ${ }^{19}$ Studies have shown that women have a higher prevalence, of psychiatric disorders related to stress throughout life, such as depression and anxiety disorders, while men have a higher prevalence of externalizing disorders, for example, aggression..$^{20}$ This suggests that men and women may have different neural resources in response to stressrelated anxiety. ${ }^{21} \mathrm{~A}$ recent study showed higher levels of anxiety, depression, and health anxiety in women during the COVID-19 pandemic. ${ }^{22}$ Anxiety disorder has been reported to be three times higher in women than in men during the pandemic. ${ }^{23}$ In addition, females were more potent predictors of post-traumatic stress disorder symptoms after pandemics. ${ }^{24}$

In this study, there was no statistically significant association between anxiety or depression symptoms and TMD symptoms. However, this association has been demonstrated in the literature, and anxiety is more associated with muscle pain and joint pain, as well as with depression. ${ }^{25}$ Depression and anxiety can contribute to TMD, interacting with pain-modulating networks, lowering the threshold, or altering the perception of pain in patients suffering from anxiety or depression, although the exact mechanism remains unclear. ${ }^{26}$ It is possible that if the sample was larger and encompassed other population profiles, such as health professionals who are working directly with the 
treatment of patients with COVID-19, and patients who are part of the risk groups, there will possibly be an association between TMD symptoms, mostly painful TMD and symptoms of anxiety and depression. However, more studies must be conducted to better understand this relationship during the period of COVID-19. It is known that the prevalence of TMD in university students is high according to data confirmed in several studies prior to the pandemic, ${ }_{1}^{27-29}$ and there are still no studies evaluating the relationship between anxiety and depression symptoms and TMD symptoms during the COVID-19 pandemic. Therefore, the results of the present study must be interpreted with caution. In addition, it is important to continue monitoring the research participants after the end of the pandemic, as according to Almeida-Leite, Stuginski-Barbosa and Conti ${ }^{30}$ (2020), the occurrence of TMD signs and symptoms after the pandemic is expected to follow a similar pattern to post-traumatic stress syndrome.

This study showed a positive association between oral behaviors and symptoms of TMD, anxiety, and depression. Chow and Cioffi ${ }^{31}$ (2019) reported an association between oral behaviors during wakefulness (clenching teeth, leaving the jaw in a rigid position, pressing the tongue against the teeth, playing with the tongue, cheeks, lips, or chewing gum) and painful TMD. These habits are more frequent in patients with painful TMD and are activities that require repeated and sustained contraction of the muscles of the jaw, which can result in muscle overload, local ischemia, and pain. ${ }^{32}$ The frequency of oral behaviors is increased in patients with greater anxiety. ${ }^{33}$ People with high levels of anxiety were shown to have a higher frequency of oral behaviors if pain was present. ${ }^{34}$ Moreover, pain increased the somatic body sensation and contributed to hypervigilance. ${ }^{31}$ Studies evaluating oral conditions with symptoms of TMD, anxiety, and depression during the COVID-19 pandemic have not been found in the literature.

The present study showed a low percentage of total social isolation (21.2\%). Possibly, this result may be associated with the profile of the public analyzed and the quarantine time. Previous studies show that high-risk patients are over 70 years old and have preexisting health problems, such as respiratory diseases, cardiovascular diseases, cancer, ${ }^{35}$ hypertension, diabetes, smoking patients, chronic obstructive pulmonary disease, and kidney diseases. ${ }^{36}$ The lower risk of complications may have resulted in relaxation concerning social isolation, with most students reporting that they were experiencing partial social isolation, leaving home at some point during the week. Another factor that may have influenced this result is the number of days of social isolation since monitoring vehicles show that the index of social isolation, over the days, has decreased. The Federal District had a rate of $65.6 \%$ on March 22, 2020, and currently (on May 30, 2020), it stands at 39.2\%. ${ }^{37}$ However, it is essential to emphasize the importance of government measures to reduce the spread of the SARS-CoV-2 virus. A study carried out in the United States of America showed that if there were no social distance measures, the COVID-19 growth rate could have been ten times higher. ${ }^{38}$

Thus, investigations about the influence of the pandemic and its consequences on TMDs should be carried out to better understand the current situation, and how it will influence the post-pandemic in orofacial pain. The major limitation of the present study was that it was carried out in a very specific population. Therefore, the results cannot be extrapolated convincingly to the general population.

\section{Conclusion}

Social isolation and stressful situations due to the COVID-19 pandemic can increase the number of people with symptoms of TMD, anxiety, and depression. Further studies should be carried out during and after the social isolation period to assess the consequences of the pandemic on psychological alterations and TMDs.

\section{Conflicts of interest}

The authors report no conflicts of interest

\section{Authors' contributions}

Medeiros, Rodrigo: Conceptualization (Equal); Data curation (Equal); Formal analysis (Equal); Funding acquisition (Equal); Investigation (Equal); Methodology (Equal); Project administration (Equal). Vieira, Danielle Leal: Conceptualization (Equal); Methodology (Equal); Project administration (Equal). Silva, Emily: Formal analysis (Equal); Writing-review \& editing (Equal). Rezende, Liliana Vicente Melo de Lucas: Conceptualization (Equal); Supervision (Equal); Writing-review \& editing (Equal). Santos, 
Rodrigo Wendel: Conceptualization (Equal); Writingreview \& editing (Equal). Tabata, Lucas Fernando: Conceptualization (Equal); Supervision (Equal); Writing-review \& editing (Equal).

\section{References}

1- Li W, Yang Y, Liu ZH, Zhao YJ, Zhang Q, Zhang L, et al. Progression of mental health services during the COVID-19 outbreak in China. Int J Biol Sci. 2020;16(10):1732-8. doi: 10.7150/ijbs.45120

2- Lu H, Stratton CW, Tang YW. Outbreak of pneumonia of unknown etiology in Wuhan, China: the mystery and the miracle. J Med Virol. 2020;92(4):401-2. doi: 10.1002/jmv.25678

3- Huang C, Wang Y, Li X, Ren L, Zhao J, Hu Y, et al. Clinical features of patients infected with 2019 novel coronavirus in Wuhan, China. Lancet. 2020;395(10223):497-506. doi: 10.1016/S0140-6736(20)30183-5 4- Xiang YT, Yang Y, Li W, Zhang L, Zhang Q, Cheung T, et al. Timely mental health care for the 2019 novel coronavirus outbreak is urgently needed. Lancet Psychiatry. 2020;7(3):228-9. doi: 10.1016/S22150366(20)30046-8

5- Brooks SK, Webster RK, Smith LE, Woodland L, Wessely S, Greenberg $\mathrm{N}$, et al. The psychological impact of quarantine and how to reduce it: rapid review of the evidence. Lancet. 2020;395(10227). doi: 10.1016/ S0140-6736(20)30460-8

6- Theroux J, Stomski N, Cope V, Mortimer-Jones S, Maurice L. A cross-sectional study of the association between anxiety and temporomandibular disorder in Australian chiropractic students. J Chiropr Educ. 2019;33(2):111-7. doi: 10.7899/JCE-18-3

7- Brancher JA, Spada PP, Meger MN, Fatturri AL, Dalledone M, Paiva Bertoli FM, et al. The association of genetic polymorphisms in serotonin transporter and catechol-O-methyltransferase on temporomandibular disorders and anxiety in adolescents. J Oral Rehabil. 2019;46(7):597604. doi: 10.1111/joor.12783

8- Natu VP, Yap AU, Su MH, Irfan Ali NM, Ansari A. Temporomandibular disorder symptoms and their association with quality of life, emotiona states and sleep quality in South-East Asian youths. J Oral Rehabil. 2018;45(10):756-63. doi: 10.1111/joor.12692

9- Bertoli FM, Bruzamolin CD, Kranz GO, Losso EM, Brancher JA, Souza JF. Anxiety and malocclusion are associated with temporomandibular disorders in adolescents diagnosed by RDC/TMD: a cross-sectional study. J Oral Rehabil. 2018;45(10):747-55. doi: 10.1111/joor.12684 10- Goncalves DA, Dal Fabbro AL, Campos JA, Bigal ME, Speciali JG. Symptoms of temporomandibular disorders in the population: an epidemiological study. J Orofac Pain. 2010;24(3):270-8.

11- Ohrbach R, editor. Critérios de diagnóstico para desordens temporomandibulares: protocolo clínico e instrumentos de avaliação. Portuguese Version 25May2016. Pereira FJ Jr, Gonçalves DA, translators. [place unknown]: International Network for Orofacial Pain and Related Disorders Methodology; 2016 [cited 2020 May 10] Available from: https://ubwp.buffalo.edu/rdc-tmdinternational/

12- Marcolino JA, Suzuki FM, Alli LA, Gozzani JL, Mathias LA. Medida da ansiedade e da depressão em pacientes no pré-operatório: Estudo comparativo. Rev Bras Anestesiol. 2007;57(2):157-66. doi: 10.1590/ S0034-70942007000200004

13- Castro MM, Quarantini L, Batista-Neves S, Kraychete DC, Daltro C, Miranda-Scippa A. Validade da escala hospitalar de ansiedade e depressão em pacientes com dor crônica. Rev Bras Anestesiol. 2006;56(5):470-7. doi: 10.1590/S0034-70942006000500005
14- Nazeri M, Ghahrechahi HR, Pourzare A, Abareghi F, Samiee-Rad $S$, Shabani $M$, et al. Role of anxiety and depression in association with migraine and myofascial pain temporomandibular disorder. Indian J Dent Res. 2018;29(5):583-7. doi: 10.4103/0970-9290.244932 15- Lei L, Huang X, Zhang S, Yang J, Yang L, Xu M. Comparison of prevalence and associated factors of anxiety and depression among people affected by versus people unaffected by quarantine during the COVID-19 epidemic in southwestern China. Med Sci Monit. 2020;26:e924609. doi: 10.12659/MSM.924609

16- Zhai Y, Du X. Addressing collegiate mental health amid COVID-19 pandemic. Psychiatry Res. 2020;288:113003. doi: 10.1016/j. psychres.2020.113003

17- Whang $Z \mathrm{H}$, Yang $\mathrm{HL}$, Yang YQ, Liu D, Li ZH, Zhang XR, et al. Prevalence of anxiety and depression symptom, and the demands for psychological knowledge and interventions in college students during COVID-19 epidemic: a large cross-sectional study. J Affect Disord. 2020;275:188-93. doi: 10.1016/j.jad.2020.06.034

18- Odriozola-González P, Planchuelo-Gómez A, Irurtia MJ, Luis-Garcia R. Psychological effects of the COVID-19 outbreak and lockdown among students and workers of a Spanish university. Psychiatry Res. 2020;290:113108. doi: 10.1016/j.psychres.2020.113108

19- Asher M, Asnaani A, Aderka IA. Gender differences in social anxiety disorder: a review. Clin Psychol Rev. 2017;56:1-12. doi: 10.1016/j. cpr.2017.05.004

20- Hickas BM, Krueger RF, Iacono WG, McGue M, Patrick CJ. Family transmission and heritability of externalizing disorders: a twinfamily study. Arch Gen Psychiatry. 2004;61(9):922-8. doi: 10.1001/ archpsyc.61.9.922

21- Seo D, Ahluwalia A, Potenza MN, Sinha R. Gender differences in neural correlates of stress-induced anxiety. J Neurosci Res. 2017;95(12):115-25. doi: $10.1002 /$ jnr.23926

22- Özdin S, Özdin SB. Levels and predictors of anxiety, depression and health anxiety during COVID-19 pandemic in Turkish society: the importance of gender. Int J Soc Psychiatry. 2020;66(5):504-11. doi: $10.1177 / 0020764020927051$

23- Wang Y, Di Y, Ye J, Wei W. Study on the public psychological states and its related factors during the outbreak of coronavirus disease 2019 (COVID-19) in some regions of China. Psychol Health Med. Forthcoming 2020. doi: $10.1080 / 13548506.2020 .1746817$

24- Liu N, Zhang F, Wei C, Jia Y, Shang Z, Sun L, et al. Prevalence and predictors of PTSS during COVID-19 outbreak in China hardest-hit areas: gender differences matter. Psychiatry Res. 2020;287:112921. doi: 10.1016/j.psychres.2020.112921

25- Kindler S, Samietz S, Houshmand M, Grabe HJ, Bernhardt O, Biffar $\mathrm{R}$, et al. Depressive and anxiety symptoms as risk factors for temporomandibular joint pain: a prospective cohort study in the general population. J Pain. 2012;13(12):1188-97. doi: 10.1016/j. jpain.2012.09.004

26- Vedolin GM, Lobato VV, Conti PC, Lauris JR. The impact of stress and anxiety on the pressure pain threshold of myofascial pain patients. J Oral Rehabil. 2009;36(5):313-21. doi: 10.1111/j.13652842.2008.01932.x

27- Alahmary AW. Association of temporomandibular disorder symptoms with anxiety and depression in Saudi dental students. Open Access Maced J Med Sci. 2019;7(23):4116-9. doi: 10.3889/ oamjms.2019.746

28- Minghelli B, Morgado M, Caro T. Association of temporomandibular disorder symptoms with anxiety and depression in Portuguese college students. J Oral Sci. 2014;56(2):127-33. doi: 10.2334/josnusd.56.127 29- Bonjardim LR, Lopes-Filho RJ, Amado G, Alburquerque RL Jr, Goncalvez SR. Association between symptoms of temporomandibular disorders and gender, morphological occlusion, and psychological factors in a group of university students. Indian J Dent Res. 2009;20(2):190-4. doi: 10.4103/0970-9290.52901 
30- Almeida-Leite CM, Stuginski-Barbosa J, Conti PC. How psychosocial and economic impacts of COVID-19 pandemic can interfere on bruxism and temporomandibular disorders? J Appl Oral Sci. 2020;28:e20200263. doi: 10.1590/1678-7757-2020-0263

31- Chow JC, Cioffi I. Effects of trait anxiety, somatosensory amplification, and facial pain on self-reported oral behaviors. Clin Oral Investig. 2019;23(4):1653-61. doi: 10.1007/s00784-018-2600-1

32- Suzuki S, Castrillon EE, Arima T, Kitagawa Y, Svensson P. Blood oxygenation of masseter muscle during sustained elevated muscle activity in healthy participants. J Oral Rehabil. 2016;43(12):900-10. doi: $10.1111 /$ joor. 12450

33- Cioffi I, Michelotti A, Perrotta S, Chiodini P, Ohrbach R. Effect of somatosensory amplification and trait anxiety on experimentally induced orthodontic pain. Eur J Oral Sci. 2016;124(2):127-34. doi: 10.1111/eos. 12258
34- Machado NA, Costa YM, Quevedo HM, Stuginski-Barbosa J, Valle $\mathrm{CM}$, Bonjardim LR, et al. The association of self-reported awake bruxism with anxiety, depression, pain threshold at pressure, pain vigilance, and quality of life in patients undergoing orthodontic treatment. J Appl Oral Sci. 2020;28:e20190407. doi: 10.1590/1678-2019-0407

35- Jordan RE, Adab P, Cheng KK. Covid-19: risk factors for severe disease and death. BMJ. 2020;368:m1198. doi: 10.1136/bmj.m1198 36- Emami A, Javanmardi F, Pirbonyeh N, Akbari A. Prevalence of underlying diseases in hospitalized patients with COVID-19: a systematic review and meta-analysis. Arch Acad Emerg Med. 2020;8(1):e35.

37- Inloco. Mapa brasileiro da COVID-19 [Internet]. Recife: Inloco; c2020 [cited 2020 Sept 11]. Available from: https:// mapabrasileirodacovid.inloco.com.br/pt/

38- Courtemanche C, Garuccio J, Le A, Pinkston J, Yelowitz A. Strong social distancing measures in The United States reduced the COVID-19 growth rate. Health Aff (Millwood). 2020;39(7):1237-246. doi: $10.1377 /$ hlthaff. 2020.00608 